\title{
Cranial MR characteristics of Cerebral Palsy cases and correlation of findings with clinical results
}

\author{
Asuman Ali' ${ }^{1}$ Ramazan Yalçın², Ayşem Ünlüer-Gümüştaş3 \\ Department of ${ }^{1}$ Neurology, Yuksek Ihtisas Training and Research Hospital, Department of ${ }^{2}$ Radiology, Bursa State Hospital; \\ Department of ${ }^{3}$ Radiology, Bursa Special Radiological Diagnostic Center, Bursa, Turkey. E-mail: asumanali@gmail.com \\ Received: 3rd April 2017, Revised: 19th August 2017, 18th September 2017, Accepted: 22nd November 2018
}

SUMMARY: Ali A, Yalçın R, Ünlüer-Gümüştaş A. Cranial MR characteristics of Cerebral Palsy cases and correlation of findings with clinical results. Turk J Pediatr 2019; 61: 525-537.

The aim of this study was to compare cranial Magnetic Resonance (MR) imaging findings in cerebral palsy (CP) patients with clinical findings and evaluate damage ascribed to prenatal, natal and postnatal reasons by preterm, term or postterm characteristics.

One thousand, one hundred CP patients registered at Dr. Ayten Bozkaya State Hospital of Cerebral Palsy Children and Rehabilitation Center from 2007 to 2010 were included in the study retrospectively. Cranial MR images studied in Bursa State Hospital Radiology Unit were evaluated by "a single senior MRI radiologist".

The most common abnormality was cerebral atrophy $(80.2 \%)$ followed by periventricular leukomalacia (PVL) (49.9\%). The most common type was spastic tetraplegia (67.9\%), and the second most common type was hypotonic CP (15.6\%). The percentage of congenital central nervous system (CNS) abnormality was $14.09 \%$. The most common abnormality was corpus callosum (CC) agenesis and colpocephaly $(2.3 \%)$. The least common conditions were cortical dysplasia, heterotopia and rhombencephalosynapsis. The percentage of cases born to consanguineous parents was 19.2\%. In Hypoxic Ischemic Encephalopathy (HIE) patients born to consanguineous parents, PVL was cystic and moderate. Consanguineous marriage was statistically significant in the patients with cortical dysplasia and heterotopia $(\mathrm{p}<0.04)$, delay in myelination $(p<0.001)$ and thalamus involvement $(p<0.008)$. Consanguineous marriage was also statistically significant in spastic diparetic $(p<0.017)$, hypotonic $(p<0.001)$ and bedridden patients $(p<0.006)$. The presence of both congenital CNS anomaly and PVL was only revealed in spastic tetraparetic type. Twentynine $\%$ of the cases were premature, while $66.4 \%$ were term children. Low birth weight $(32.7 \%)$ constituted a risk factor for all clinical types, except mixed type CP. The percentage of patients with natal causes only was $21.5 \%$. Kernicterus, cerebrovascular causes and HIE accounted for $6.3 \%, 2.9 \%$ and $54.4 \%$ of the cases, respectively.

This study may indicate the importance of consanguineous marriage, natal and postnatal care properties as the underlying causes of CP beyond the clinical management related to delivery and allow us to identify strategies for possible measures.

Key words: cerebral palsy, cranial Magnetic Resonance Imaging, consanguineous parents, congenital CNS abnormality. 
Cerebral palsy (CP) is the most common cause of physical disability in childhood yet in many cases the cause remains unknown. The term "cerebral palsy" describes a group of disorders of movement and posture due to a defect or lesion of immature brain. ${ }^{1}$ In an international workshop in 2007, results on classification and description of $\mathrm{CP}$ were published. ${ }^{3} \mathrm{~A}$ developing brain may suffer damage for prenatal, natal or postnatal reasons.,3 Other major risk factors include prematurity and low birth weight. Injury may be associated with congenital, genetic, inflammatory, infectious, anoxic, traumatic and metabolic reasons. ${ }^{4,5}$ Reported worldwide incidence of CP is about 2 to 2.5 cases per 1000 births. $^{5-7}$ This rate varies in female and male children . A large European dataset of 4500 children with CP found that the incidence of CP was $30 \%$ higher in males than females. ${ }^{8}$ For the past four decades, the prevalence and incidence of CP have remained constant despite the technological advances decreasing mortality in both term and preterm infants. ${ }^{9}$

The diagnosis of CP is based on the person's history and physical examination. However, magnetic resonance imaging (MRI) especially has the potential to identify the lesion or abnormality and thus can help to determine the timing of CP origin. MRI, which allows differentiation between gray and white matter in the brain and shows resolution of corticalsubcortical infarcts, atrophy or injuries, enables identification of central nervous system congenital anomalies, thus determination of prognosis of a patient and enables clinicans to carry out genetic tests accordingly. Cranial MRI not only reveals pathology but also shows results enabling correlation with clinical findings. ${ }^{10-15}$ When systematically analyzing studies using MRI in children with CP, MRI proves to be abnormal in around $85 \%$ of cases and gives a clear pathogenetic pattern in more than $80 \%$. The harmonized classification of MRI, is based on pathogenic patterns MRI classification system (MRICS) proposed by the Surveillance of Cerebral Palsy in Europe (SCPE). SCPE proposes the MRICS as a reliable tool. ${ }^{13}$

Several lines of evidence support the theory that multiple genetic factors contribute to the cause of CP. Tollanes et al. ${ }^{14}$ argue that CP has a genetic component and the higher the risk of its recurrence among relatives, the closer the genetic relationship. The risk of $\mathrm{CP}$ in consanguineous families is about 2-5 times higher than the risk in outbred families. ${ }^{15,16}$ The rate of epilepsy in children with CP is strikingly higher, ranging from $15 \%$ to more than $60 \%$, depending on the type of $\mathrm{CP}^{17,18}$

Among the important known causes of $\mathrm{CP}$ are congenital brain malformations including malformations of cortical development. Modern imaging techniques enable more children with these conditions to be identified ${ }^{19}$ and knowledge about the cortical dysplasias, of which some have a genetic basis, is increasing rapidly. ${ }^{19-25}$ Risk of having a child with disability in unrelated parents is $2-3 \%$, in first cousins is $4-5 \%$ and double first cousins is $6-7 \% .{ }^{19-22}$

Yin et al. ${ }^{21}$ evaluated cranial MR imaging findings of a series of 42 cases diagnosed with $\mathrm{CP}$ and found abnormalities in 39. In a study reported by the European Cerebral Palsy study group, a total of 351 children were evaluated, and abnormal MR characteristics were shown in 310 patients $(88.3 \%) .{ }^{23}$ KrägelohMann and Horber's population-based study ${ }^{12}$ demonstrated abnormal MRI findings in 334 $(86 \%)$ of 388 patients. ${ }^{22-24}$

The objective of this study was to systematically compare the results of cranial MR imaging in CP with clinical findings and evaluate brain malformations, structural defects and damage ascribed to prenatal, natal and postnatal reasons by preterm, term or postterm characteristics. We aimed to reveal more information about the causes of $\mathrm{CP}$ by using MR imaging. We hypothesized that the clinical prognosis can be determined according to the results of MRI in the cases of CP with consanguineous families.

\section{Material and Methods}

$\mathrm{CP}$ patients aged in the range of 1 to 18 years with available hospital records, protocols and files who presented to the State Hospital of Cerebral Palsy Children and Rehabilitation Center and those who were evaluated by the same neurologist from 2007 to 2010 were included in the study, retrospectively. 
The study was done by scanning files from archive records in the same hospital. The age of the child, clinical history obtained from the child's mother and the research done to obtain information and make a diagnosis were fulfilled in all the results of clinical classification. Although lesions, abnormality or damage emerging during fetal or newborn period as well as early phases of development are stable, the emergence of clinical features may vary over time in line with maturation of central nervous system, the plasticity of growth and development. Therefore, the same neurologist performed periodic follow-up examinations of all the patients throughout the rehabilitation period. Motor weakness was defined as a score of less than or equal to four of five on the Medical Research Council Scale for Muscle Strength in at least one limb associated with activity limitation. In addition, $\mathrm{CP}$ cases with epilepsy were evaluated by the same neurologist.

Cranial MR images of the same patients studied in two specific centers (State Hospital Radiology Unit and Special Radiological Diagnostic Center) were evaluated by two radiologists working on children in a university hospital for three years. Radiological findings obtained were compared with clinical findings. For each of the cases, pregnancy and birth histories obtained particularly during interviews made with the respective mother, developmental characteristics and hospital discharge records related to past diseases were considered, and prenatal, natal and postnatal etiological causes that developed in two years were evaluated. Correlation of cranial MR findings with clinical results were based on these principles.

GE Sigma 1.5 Tesla (GE Medical Systems, USA) was used in cranial MR studies. Routine axial FLAIR (TR 8800, TE 100, TI 2200), axial T2-weighted (TR 4000, TE 100) and axial T1-weighted (TR 540, TE 9) and also sagittal T2-weighted (TR 4000, TE 100) and coronal T2-weighted (TR 4000, TE 100) study was performed.

In our study, all radiological examinations consisted of MRI scans. All of the patients aged in the range of 1 to 18 years who underwent cranial MR evaluation and had been diagnosed with CP up to postnatal two years of age were included. The patients with specified metabolic disease and those with observed progression in their periodic neurological assessments were not included in the study. File records were evaluated by taking account the criteria for the diagnosis of CP. If more than one cranial MR imaging study was done on a particular child during clinical follow-up, the last imaging study was given precedence.

The research protocols have been approved by the local ethics committee. Local ethics committee: Training and Research Hospital Clinical Research Ethics Committee (March 16, 2014; Decision Number: 2014/08/05)

\section{Statistical Analysis}

Descriptive statistics for continuous variables of the patients were given as mean and standard deviation in parametric data. Categorical data were expressed in percentage (\%) and frequency among the groups. IBM SPSS version 21 (IBM Acquires SPSS Inc., Somers, NY, USA) software package was used to analyze the data, and the Pearson chi-square test was studied in the analysis of categorical data. $\mathrm{p}<0.05$ was considered significant for the results in all statistical analyses.

\section{Results}

Cranial MR images studied in the age range of 1 to 18 years of 1100 (eleven hundred) patients diagnosed with CP from 2007 to 2010 were evaluated. Of these patients, 636 were male $(57.8 \%)$ and 464 were female $(42.2 \%)$. The number of male patients was superior to the number of female patients by $27 \%$. The mean age was $6.96 \pm 5.55$. Birth weight was $2740.75 \mathrm{~g} \pm 838.12$.

The neuroimaging results (Group I) were evaluated by MRICS

\section{Maldevelopments (Fig. 1.)}

A.1. Disorders of cortical formation: Schizencephaly (16; 1.5\%); Lissencephaly (agyria, diffuse pachygria) (14; 1.3\%); Pachygria, unilateral or bilateral (14;1.3\%); Pachygria-Polymicrogyria Complex (10; $0.9 \%)$; Focal polymicrogyria-Pachygria (6; $0.5 \%)$; Cortical dysplasia and heterotopia (1; $0.09 \%$ ). (Fig. 2 and 3), 


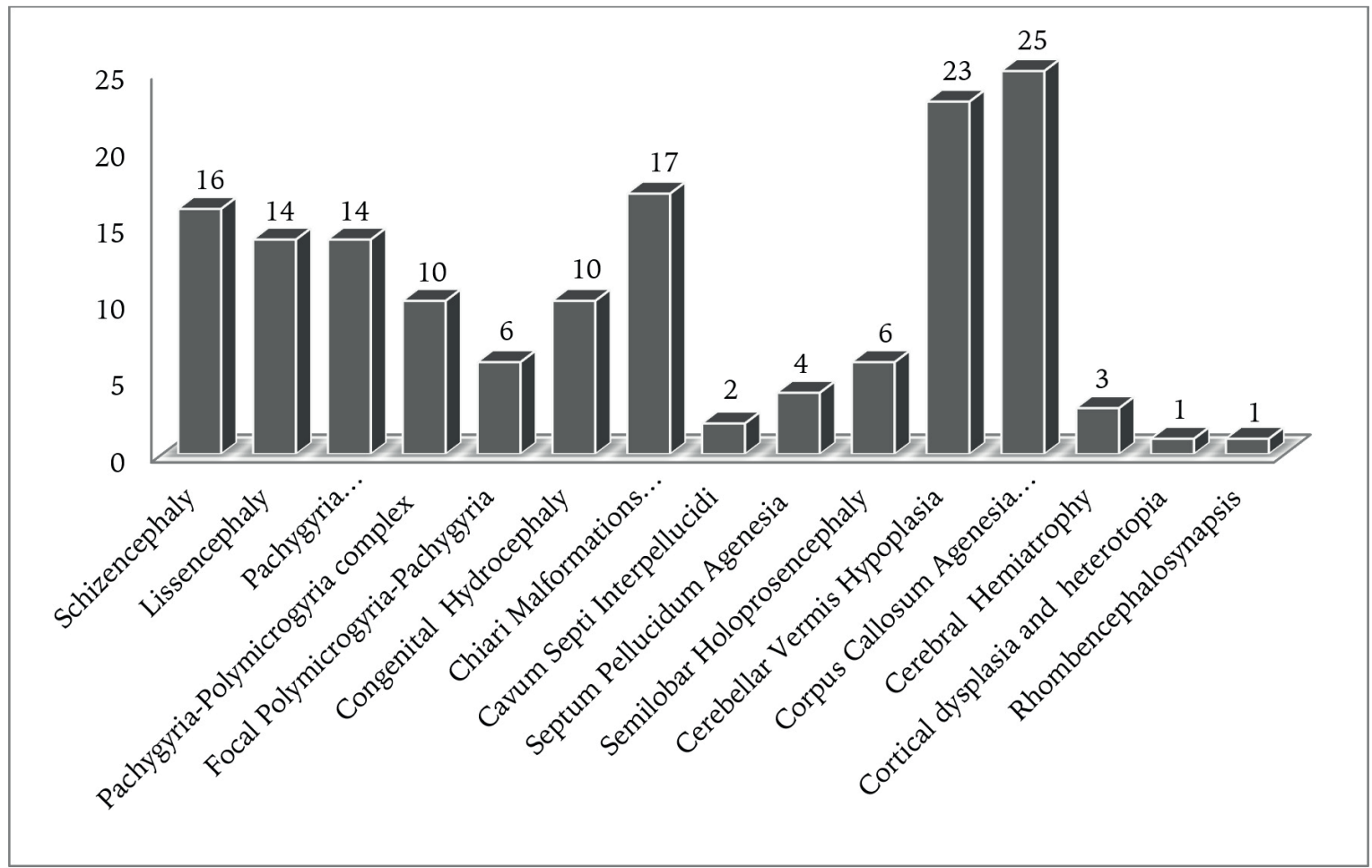

Fig. 1. Brain malformations patient Number:152

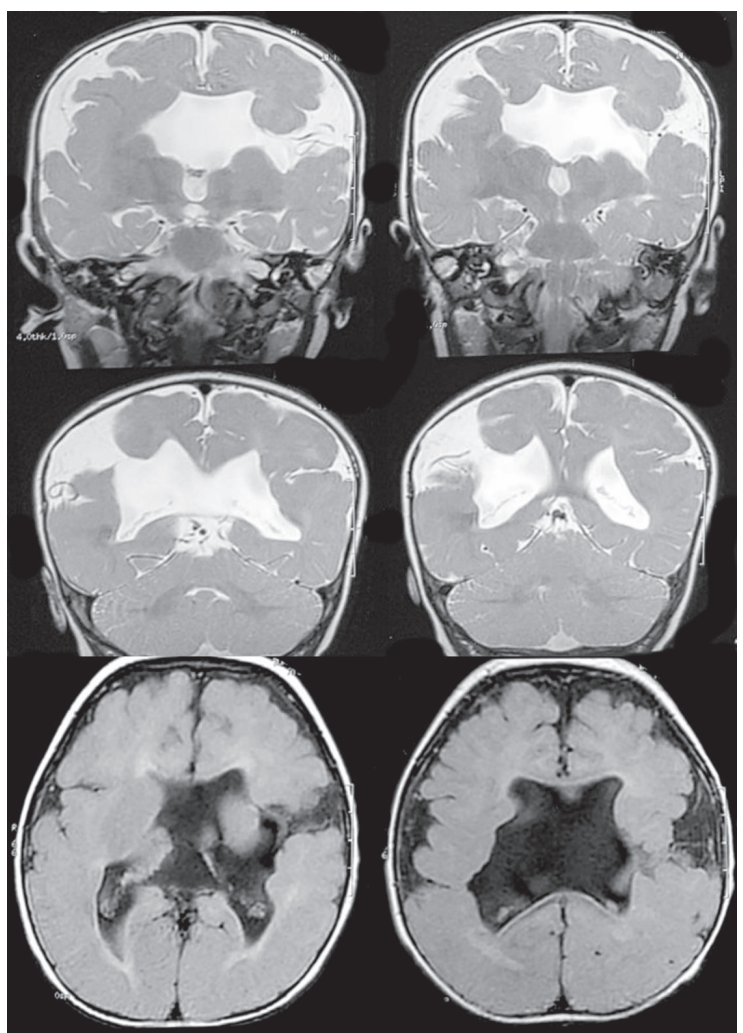

Fig. 2. Schizencephaly

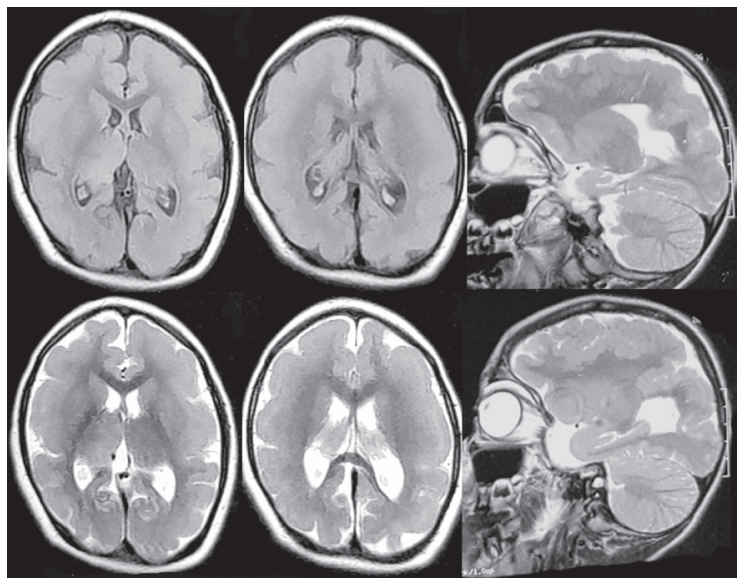

Fig. 3. Pachygria

A.2. Other maldevelopments: Congenital Hydrocephalus (10;0.9\%); ChiariMalformation (17; 1.5\%); Cavum Septi Interpellucidi (2; $0.2 \%)$; Septo-optic dysplasia or Septum Pellucidum Agenesis (4; 0.4\%); Semilobar Holoprosencephaly (6; $0.5 \%)$; Cerebellar Vermis Hypoplasia $(24 ; 2.18 \%)$; Full or partial CC (Corpus Callosum) Agenesis, Colpocephaly (25; $2.3 \%)$; Cerebral Hemiatrophy (3; $0.3 \%)$, Rhombencephalosynapsis (1;0.09\%). (Fig. 4) 
Predominant white matter injury

B.1. Periventricular leukomalacia (PVL), (549; 49.9\%); Mild, 142 (12.9\%); moderate, 371 (33.7\%); Severe, 36 (3.3\%) (Fig. 5)

B.2. Cystic Periventricular Leukomalacia (204; $18.5 \%)$

Predominant grey matter injury

C.1. Basal ganglia involvement $(186 ; 16.9 \%)$

Thalamus involvement $(242 ; 22 \%)$; Basal ganglia + thalamus exposure $(91 ; 8.3 \%)$

C.2. Corticosubcortical atrophy $(371 ; 36.4 \%)$; CC Atrophy $(517 ; 50 \%) ;$ Multicystic

Encephalomalacia $\quad(35,3.43 \%)$;Parasagittal Cerebral Injury $(35 ; 3.2 \%)$.(Fig. 6 and 7 )

\section{C.3.Arterial infarction}

D. Miscalleneous: Cerebral atrophy (871; $85.4 \%)$; Cortical atrophy $(362 ; 35.5 \%)$; Diffuse parenchymal atrophy (138; 13.5\%); Delayed myelination (93; 8.5\%); Mesial Temporal Sclerosis (7; 0.68\%) ; Hippocampal Atrophy (21; $2.05 \%)$; Brainstem involvement (109; 9.9\%) ; Cerebellum (108; 9.8\%). (Fig. 8 and 9)

E. Normal MR (80;7.3\%)

Group II was classified as follows: 1 . Consanguineous marriage $(211 ; 19.2 \%) 2$. Preterm $(319 ; 29 \%)$, Term $(730 ; 66.4 \%)$ and Postterm (51; 4.6\%) birth history 3. Low Birth Weight $(360 ; 32.7 \%) 4$. Twin or triplet pregnancies $(72 ; 6.5 \%) 5$. Hypoxic Ischemic Encephalopathy (HIE) (598; 54.4\%) 6. Prenatal (216; 19.6\%), Natal (236; 21.5\%) and Postnatal $(140 ; 12.7 \%)$ Characteristics 7. Kernicterus $(69 ; 6.3 \%)$ 8. Clinical Types of Cerebral Palsy; Spastic diplegia (168; 15.3\%), spastic hemiplegia $(103 ; 9.4 \%)$ and tetraplegia $(475 ; 43.2 \%)$; dyskinetic type $(91 ; 8.3 \%)$, ataxic $(33 ; 3 \%)$, hypotonic $(172 ; 15 \%) .6 \%)$ and mixed type $(58 ; 5.3 \%)$ and 9. Severe bedridden Cerebral Palsy (236; 21.5\%) (Fig. 10) and (Fig. 11).

Evaluation was made up to the age of 18 years according to the results obtained with The Gross Motor Function Classification System for CP- Expanded \& Revised, and 21.5\% (236) of patients were identified as severe bedridden CP (level 5).

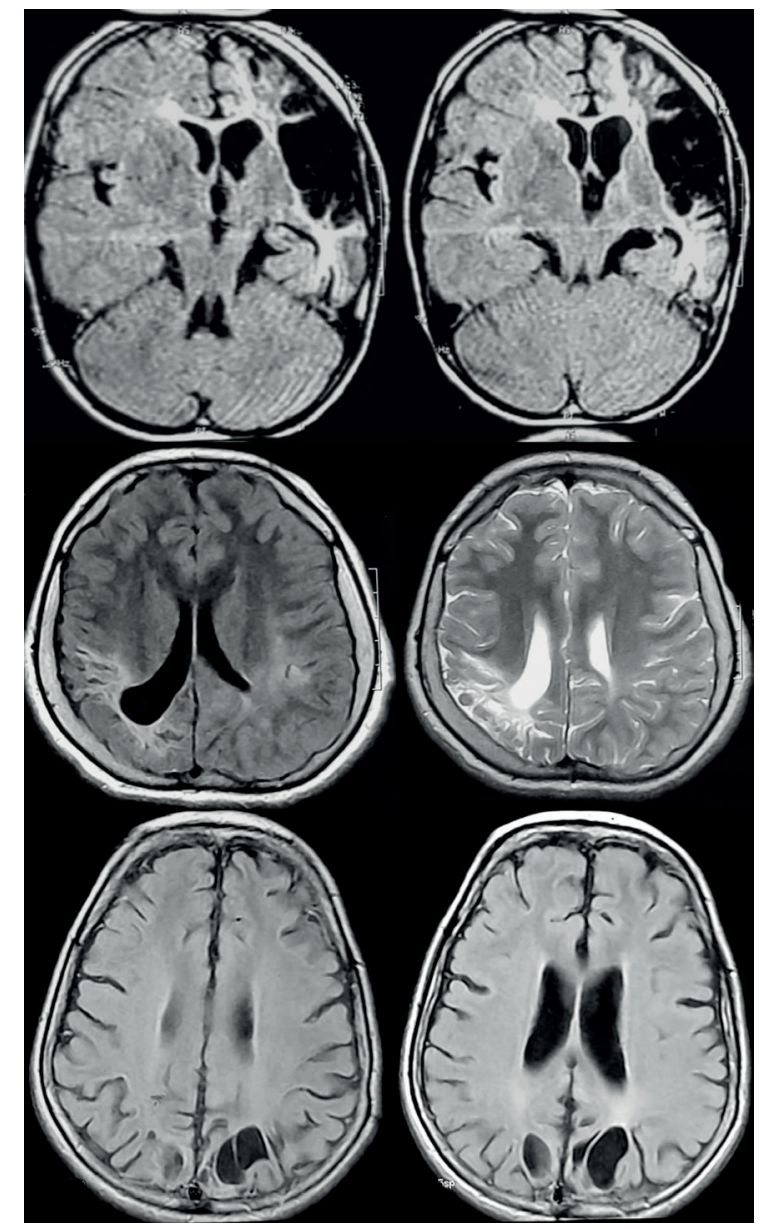

Fig. 4. Focal hemispheric injury

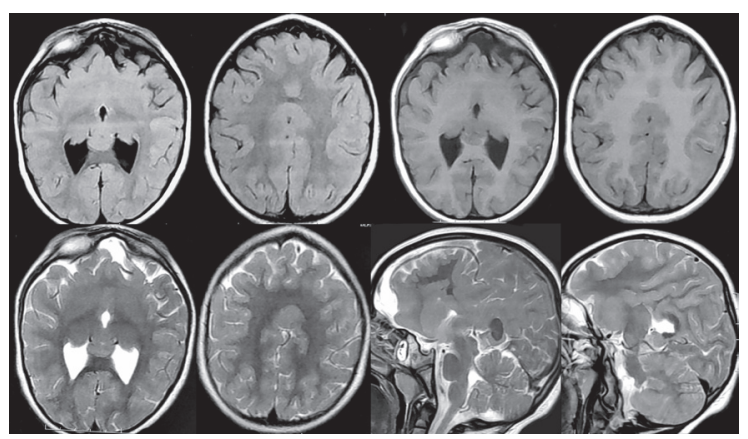

Fig. 5. Holoprosencephaly

There was statistical significance between epilepsy and corticosubcortical atrophy, hippocampal atrophy and multicystic encephalomalacia ( $p<0.04)$. One hundred and fifty-two patients $(13.8 \%)$ had congenital central nervous system (CNS) abnormalities. The most common congenital CNS abnormality was CC Agenesis and Colpocephaly (25; $2.3 \%)$. The least common conditions were 


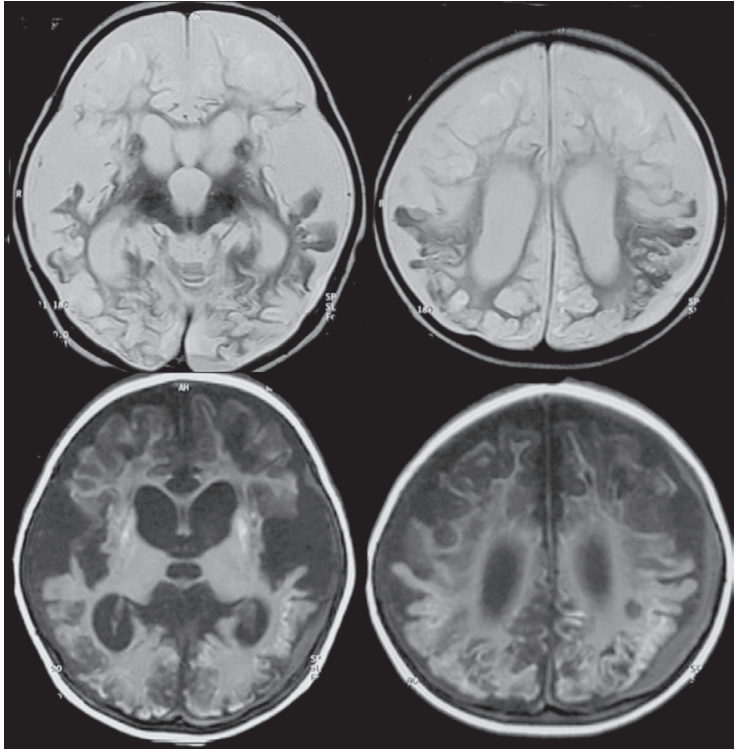

Fig. 6. Multicystic encephalomalacia

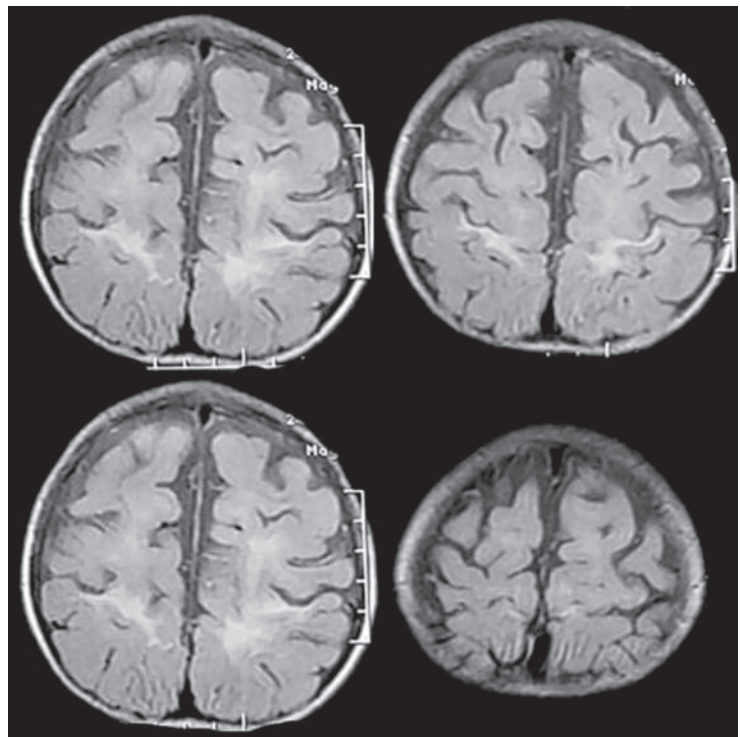

Fig. 7. Parasagittal cerebral injury

cortical dysplasia and heterotopia (1;0.09\%) and rhombencephalosynapsis ( $1 ; 0.09 \%)$ observed in a single patient. Only 9 of 16 Schizencephaly cases had Schizencephaly only. Three of the remaining 7 Schizencephaly cases also had pachygria, 3 had heterotopia and 1 had polymicrogyria. 211 (19.2\%) cases were born to consanguineous parents. Consanguineous marriages were in the form of first-degree consanguineous marriages (marriages between children of paternal uncles, marriage with son of maternal uncle or daughter of paternal aunt etc.). Cortical dysplasia and heterotopia,

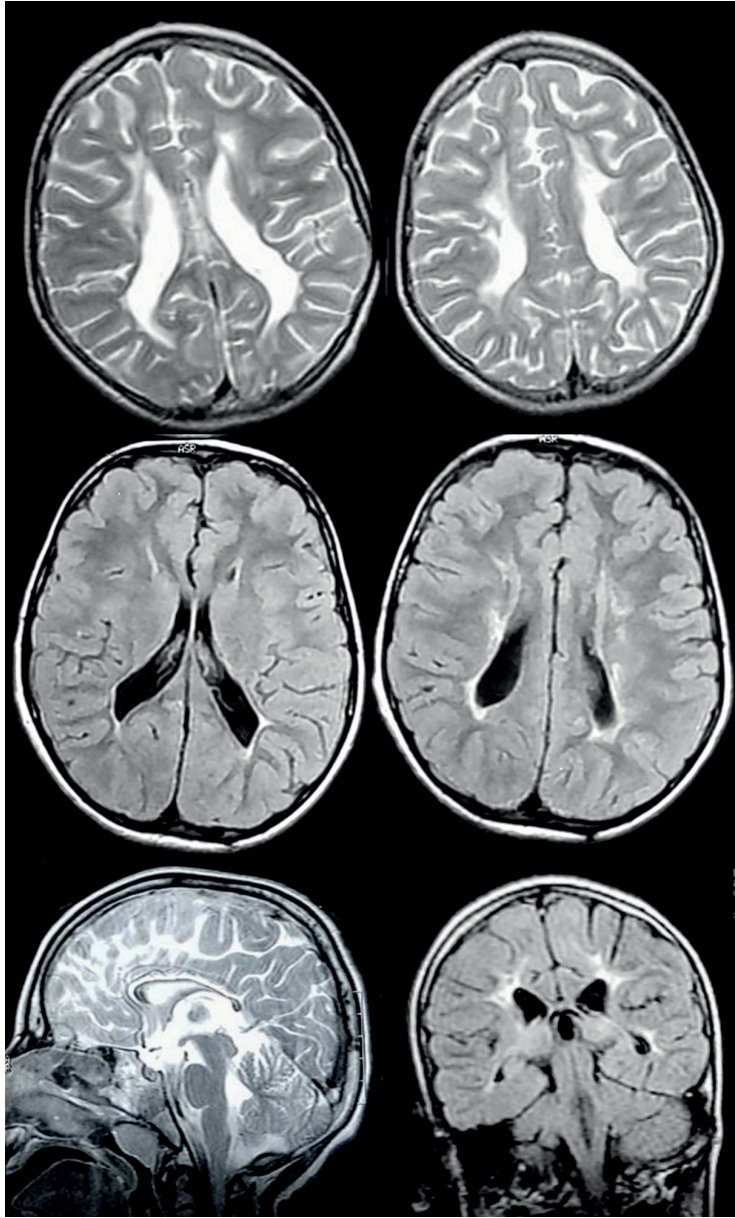

Fig. 8. Periventricular leukomalacia

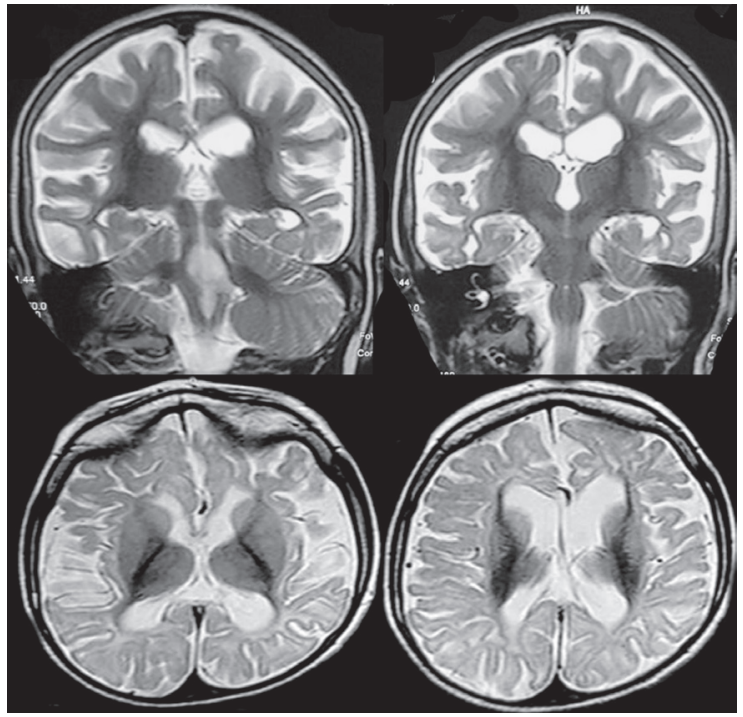

Fig. 9. Diffuse cerebral atrophy

which are congenital abnormalities, showed statistically significant correlation with consanguineous parents $(\mathrm{p}<0.04)$. Cranial 


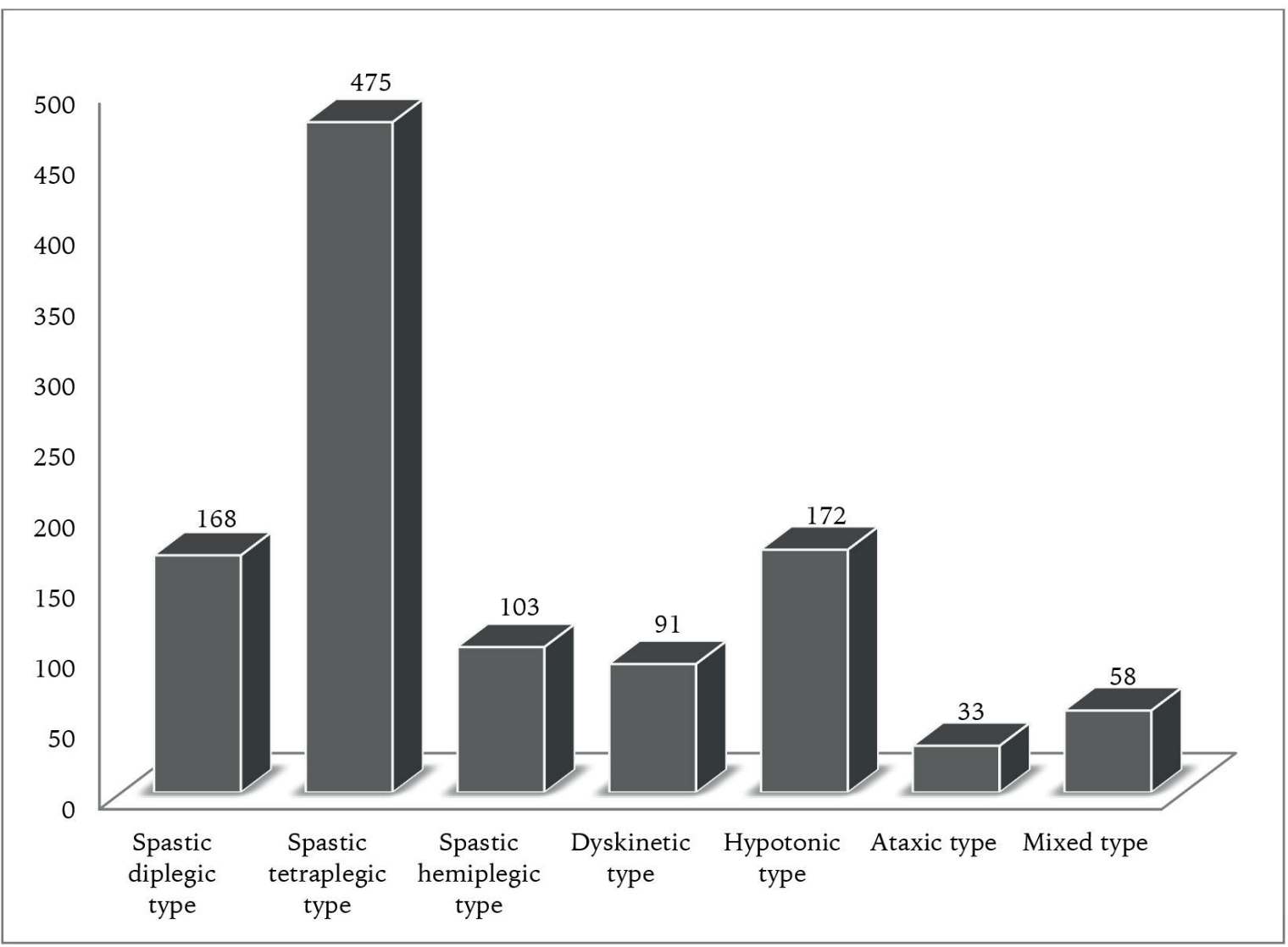

Fig. 10. Cerebral palsy subtype patient Number:1100

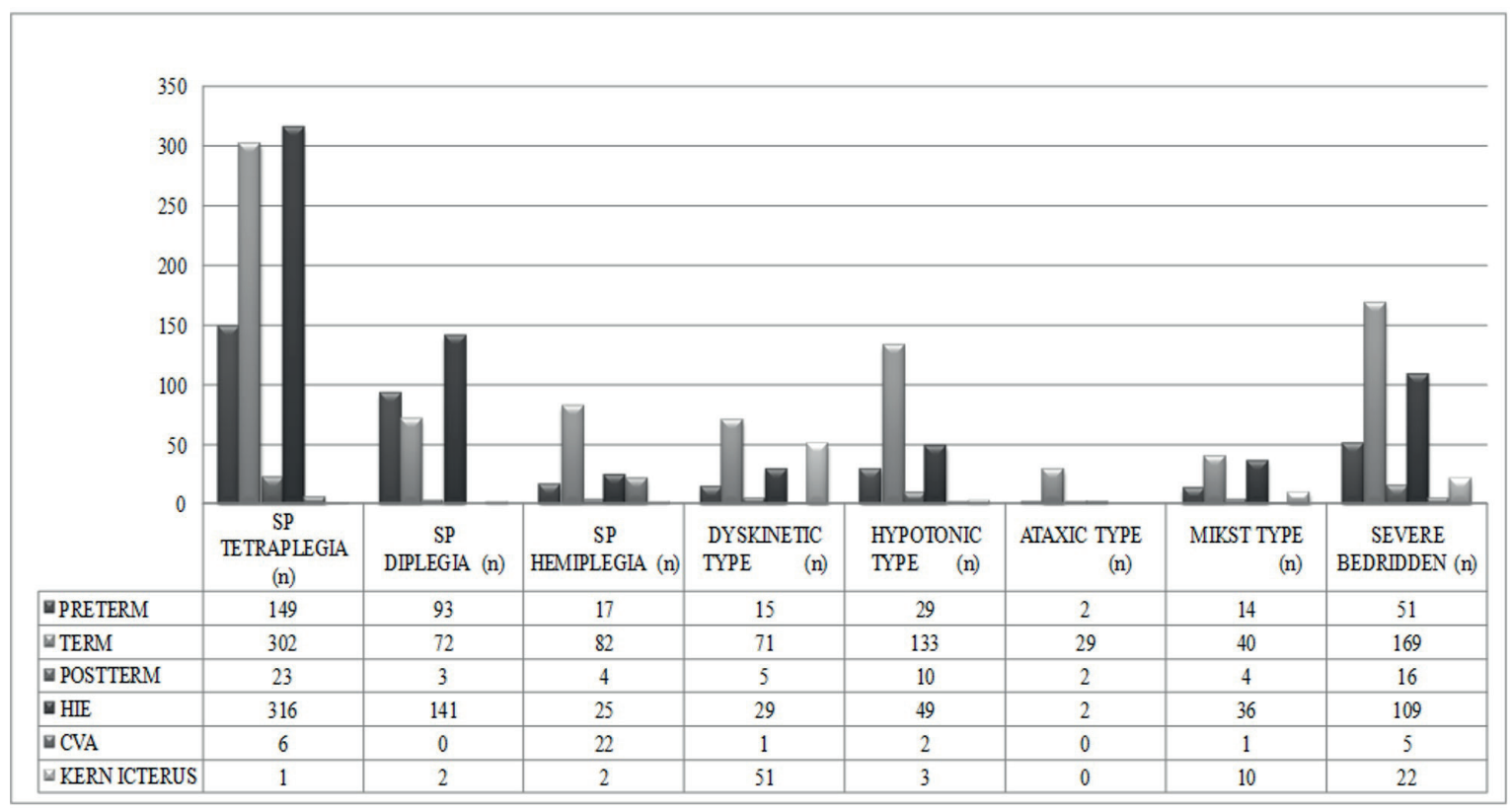

Fig. 11. Cerebral palsy: n (1100 patients) 
MRI was normal in dyskinetic and hypotonic type CP ( $\mathrm{p}<0.001)$.

The presence of both $\mathrm{CP}$ and congenital CNS abnormality was significant in other clinical types except spastic hemiplegia. Severe bedridden patients, spastic tetraplegia and diplegia patients had multicystic encephalomalacia ( $p<0.001)$. Delay in myelinization was revealed in patients with hypotonic $\mathrm{CP}$, hemiplegic $\mathrm{CP}$ and those born to consanguineous parents ( $p<0.001)$. Parasagittal cerebral damage was significant in mixed type CP $(\mathrm{p}<0.015)$ and spastic tetraplegic $(\mathrm{p}<0.016)$ patients. Thalamus involvement was only significant in spastic tetraplegic $(\mathrm{p}<0.001)$ type and patients born to consanguineous parents $(\mathrm{p}<0.008)$ as well as ataxic $(\mathrm{p}<0.002)$ type. Basal ganglion damage was only observed in mixed type and dyskinetic CP. Patients born to consanguineous parents included hypotonic $(\mathrm{p}<0.017)$, spastic diplegia $(\mathrm{p}<0.001)$ and bedridden $\mathrm{CP}$ cases $(p<0.006)$.

PVL + CC atrophy was detected in all clinical types, except spastic diplegia and tetraplegia. The presence of both congenital CNS anomaly and PVL was only revealed in spastic tetraplegic type (Table I). In our series, male children were affected more than girls by $27.1 \%$. Twenty-nine $\%$ of all CP cases were premature, while $66.4 \%$ were term children. In our study, the percentage of low birth weight was $32.7 \%$. Particularly, hypotonic CP cases were term patients. Preterm patients included bedridden ( $\mathrm{p}<0.005)$, hemiplegic ( $\mathrm{p}$ $<0.008)$ and ataxic $(\mathrm{p}<0.004)$ patients. Of our patients, $19.2 \%$ were born to consanguineous parents. In our cases, there was a statistically significant feature for CP between multiple pregnancies and term and preterm groups. Of the cases, 730 had term $(66.4 \%), 319$ had preterm (29\%) and 51 had postterm (4.6\%) history. The number of multiple pregnancy as twin or triplet pregnancy was $72(6.5 \%)$. One thousand and twent-eight $(93.5 \%)$ cases were born from singleton pregnancy. The number of CP cases born with low birth weight $(<2500$ g) was $360(32.7 \%)$. Kernicterus sequel was detected in $69(6.3 \%)$ cases. Five hundred and nighty eight patients $(54.4 \%)$ had a history of HIE and 32 patients $(2.9 \%)$ had a history of cerebrovascular disease (Table II).
Multiple pregnancies $(\mathrm{p}<0.001)$ and in vitro fertilization $(\mathrm{p}<0.001)$ appeared with spastic diplegic type. Low birth weight constituted a risk factor for all clinical types, except mixed type. In the case of bedridden patients, when natal, postnatal causes or prenatal and postnatal causes were together, significant statistics were obtained.

\section{Discussion}

$\mathrm{CP}$ is a life-long, permanent and unchanging movement, posture and motor function disorder. Its causes are not based on a single etiology. ${ }^{1} \quad$ In $\mathrm{CP}$ patients, determination of pathology and its time of development by cranial MR imaging provides reliable information about the prognosis and management of the disease..$^{9-12}$ Ashwal et al. ${ }^{2}$ studied data involving 644 children with CP who received an MRI and found abnormalities in $89 \%$ (range 68-100 \%) with the yield dependent on the type of CP present, whether the child was born preterm or at term, or if the CP was due to an insult later in life. In our series, 92.7\% abnormal images were identified and the most common finding was atrophy with $85.4 \%$. The second most common finding was periventricular white matter damage (PVL, periventricular leukomalacia) with a rate of $49.9 \%$, and the percentages of cystic PVL and severe PVL demonstrated were $18.5 \%$ and $3.3 \%$, respectively. The third most common finding was (47\%) CC atrophy, and the percentage of the presence of both PVL and CC atrophy as $34.5 \%$. A systematic review of the literature by Krägeloh-Mann et al. ${ }^{11-12}$ reported abnormal MR findings for $86 \%$ of the patients, and imaging findings yielded clues about pathogenesis by $83 \%$. In particular, they found MRI abnormalities in $90 \%$ of spastic tetraplegic and diplegic CP patients. The most common lesion was PVL (60\%). In our series, PVL was the second most common abnormality, and PVL was detected at slightmoderate level (46.6\%), except prematurity. Different subtypes of CP may be ascribed to a variety of reasons. The significant finding in patients with spastic diplegia was PVL in our series. Korzeniewski et al. ${ }^{10}$ assessed neuroimaging studies of CP from 1975 to 2006 using SCI (Science Citation Index) and Medline (OCLC) electronic indexes and 


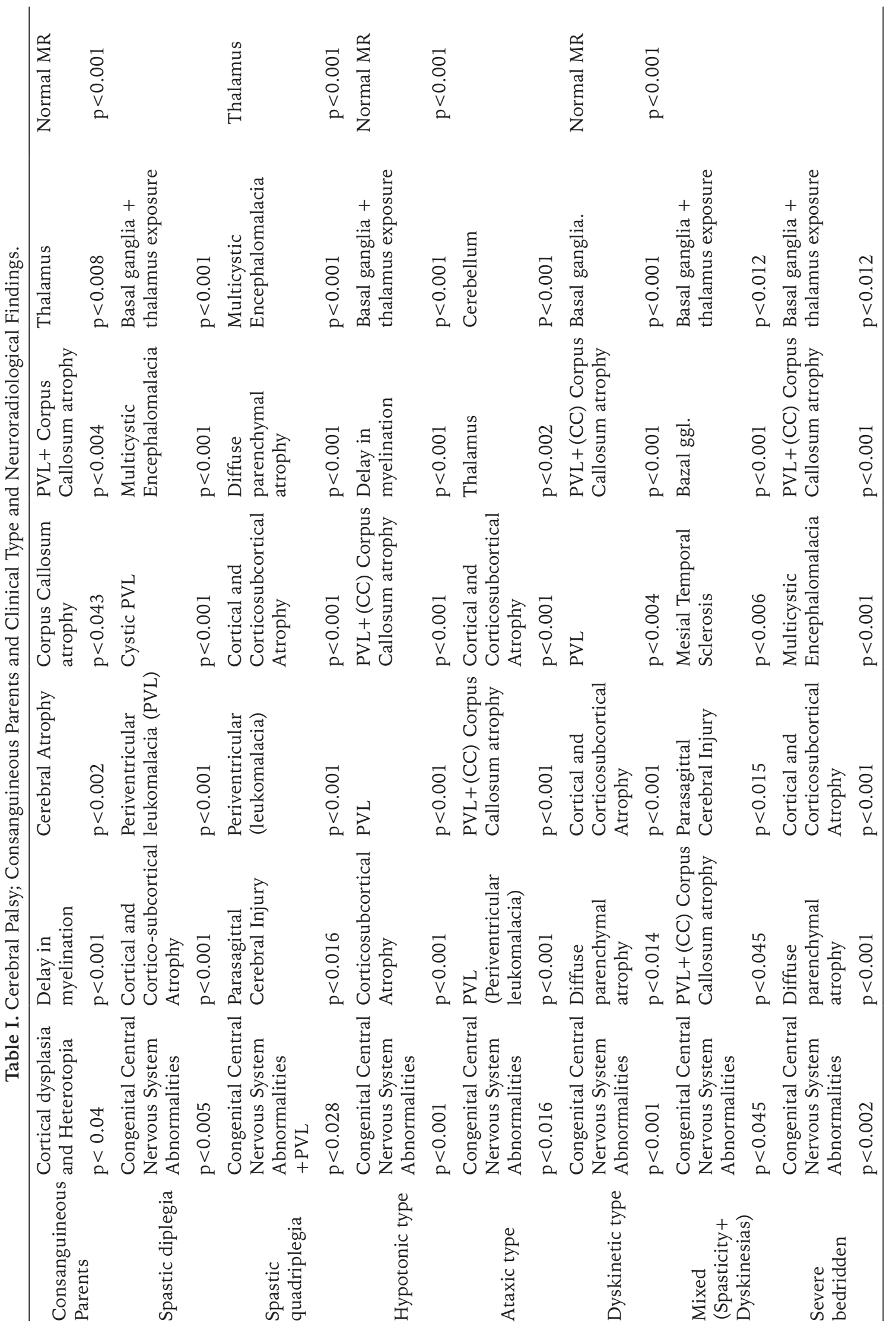




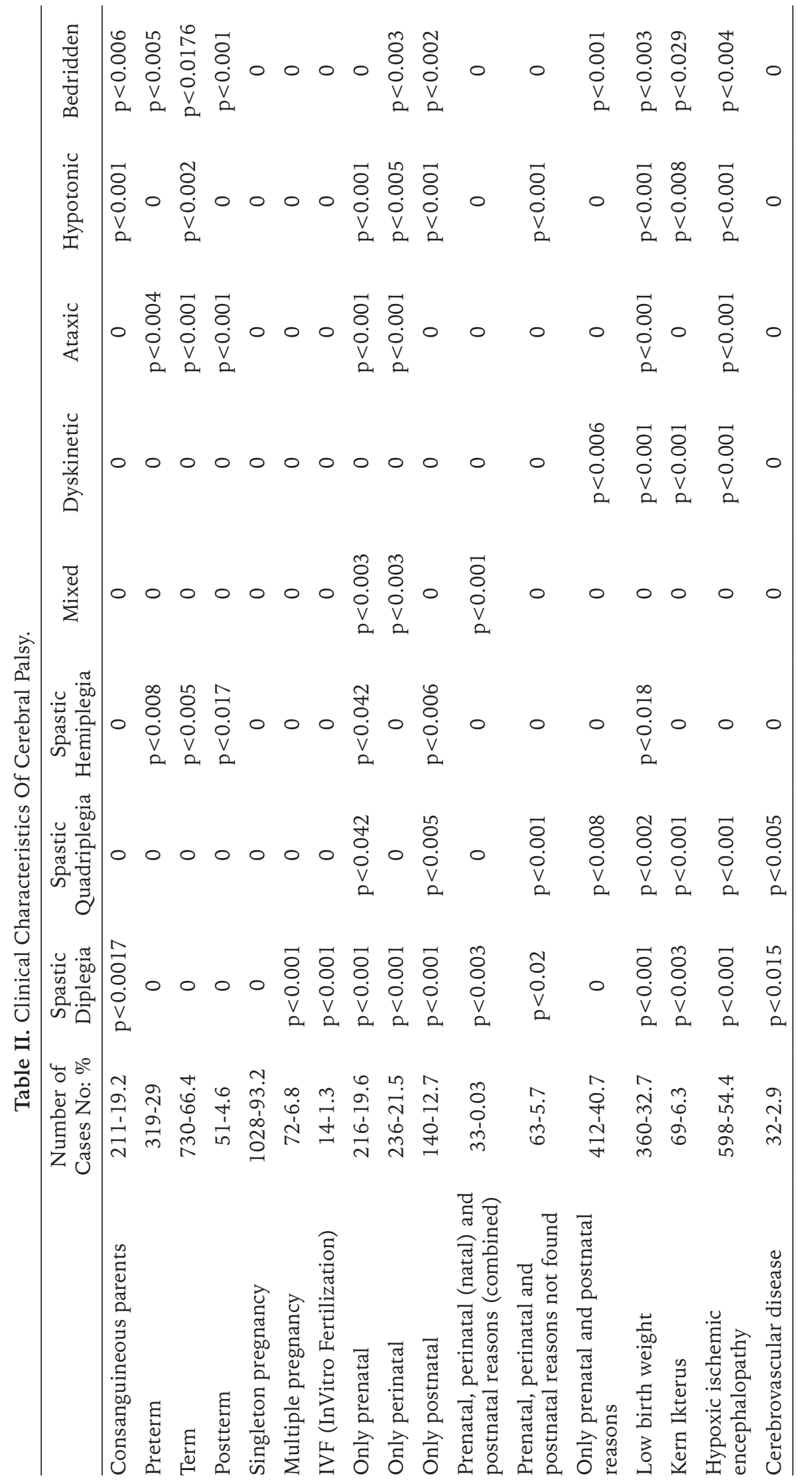


revealed $80 \%$ image abnormalities in 20 studies covering various forms of CP. White matter damage, ventriculomegaly and atrophy were the most common findings. The least common finding was isolated gray matter damage. Krägeloch-Mann et al. ${ }^{11,12}$ reported cortical or deep gray matter lesions as the second most common pattern of CP. A hospital-based study reported basal ganglia/thalamic lesion in $23 \%$ of children. In our study, the percentage of patients with thalamus lesion only was $22 \%$, the percentage of patients with basal ganglia lesions was $16.9 \%$, and the percentage of patients with both basal ganglia and thalamus lesions was $8.3 \%$. In our series, PVL and cystic PVL were shown to be particularly related to low birth weight and HIE. In HIE patients born to consanguineous parents, PVL was cystic and moderate. Previous researchers demonstrated that cortico-subcortical areas or deep gray matter (basal ganglia and thalamus) may suffer exposure during term birth or postnatal period and be damaged because of problems in late 3rd trimester. ${ }^{12,13}$ In our series, corticosubcortical exposure was present in all types of clinical CP, except mixed type CP. It was also significant in hypotonic type CP patients, to whom prenatal, natal and postnatal causes are not applicable.

Cerebellum was affected particularly in ataxic $\mathrm{CP}$ patients with history of preterm, term and postterm birth. Cerebellar exposure, a preterm damage pattern, has started to be recognized increasingly upon the use of MRI.

Our study was a retrospective study particularly based on hospital records. Patients and hospital records were found to be satisfactory because the patients were closely monitored on a periodic basis. The patients were examined by the same neurologist throughout the rehabilitation and the patients showing progression were selected and excluded from the study, which provided appropriate data. However, neonatal period records of the patients with neonatal encephalopathy or HIE were obtained from discharge records and the process of incubator care wasn't observed in situ, and these may be considered as limitations of the present study. HIE was significant in our spastic diplegic patients with a history of preterm birth. In tetraplegic CP patients, HIE was observed in patients with a history of term birth, rather than patients with a history of preterm birth. Only prenatal and/or only postnatal causes were accountable. Our spastic hemiplegic cases only had HIE arising from postnatal preventable causes. HIE was also significant in our severe bedridden $\mathrm{CP}$ patients according to Gross Motor Function Classification System - Expanded and Revised (GMFCS-ER). In our patients, the overall prevalence of epilepsy was $44.5 \%$. Patients with spastic tetraplegic CP had a higher incidence of epilepsy (\%78.2). In these children, epilepsy is an index of the severity of CP. ${ }^{17,18}$ In addition, epilepsy cases have statistical significance in cases of corticosubcortical, hypocampal atrophy and multicystic encephalomalacia. In our series, the proportion of cases with undetermined etiology was only $5.7 \%$ (63). Martin Bax et al. ${ }^{23}$ and Ingeborg Krägeloh-Man et al. ${ }^{11,12,19}$ reported that it's likely that unspecified genetic or metabolic causes account for CP in less than $10 \%$ of children.

According to studies that correlated pathological and radiological findings, brain damage patterns in $\mathrm{CP}$ are closely associated with types of CP. Many types of $\mathrm{CP}$ are classified, and the dominant type is spastic CP. Spastic CP accounted for $70-80 \%$ of all CP cases.

In our $\mathrm{CP}$ cases, the most common type was spastic tetraplegia, and the second most common type was hypotonic CP. In our study, the rate of spastic CP was $67.9 \%$ with the rates (\%) of tetraplegia, spastic diplegia and hemiplegic $\mathrm{CP}$ being 43.2, 15.3 and 9.4, respectively. In previous studies, dyskinetic (choreoathetoid and dystonic) cases comprised $10-15 \%$ of patients with $\mathrm{CP}$ and ataxic patients comprised $5 \%$ of patients with $\mathrm{CP}$, which were $8.3 \%$ and $3 \%$, respectively in our study. In our patients with a history of HIE that developed ataxia, other than cerebellum, basal ganglion $(\mathrm{p}<0.03)$ and thalamus $(p<0.001)$ involvement were found to be significant. Ataxic CP is defined as a subtype of $\mathrm{CP}$ that shows the lowest prevalence. ${ }^{4,11}$ The proportion of hypotonic CP subtype was $15.6 \%$ in our study. In a study conducted in Norway by Tollånes et al. ${ }^{14}$ the risk of $\mathrm{CP}$ was increased 6-9 fold in first degree relatives, 3-fold in second degree relatives, and 1.5 -fold in third 
degree relatives. Tollånes et al. ${ }^{14}$ suggested that $\mathrm{CP}$ has a genetic component, that the higher the risk of repeat among relatives, the closer the genetic relationship, and they concluded that the underlying causes of $\mathrm{CP}$ extend beyond the clinical management of delivery. In our series, the patient with identified cortical dysplasia and heterotopia was born to first degree consanguineous parents and this result was statistically significant $(\mathrm{p}<0.04)$. The same findings were established between consanguineous parents and thalamus involvement and delay in myelination. Having consanguineous parents was also significant for bedridden patients with a severe CP clinic course in the case of hypotonic CP and for patients with diffuse parenchymal atrophy as revealed by their MR images. KrägelohMann I et al. ${ }^{11,12,19}$ reported the percentage of genetic or acquired brain malformations as approximately $10 \%$. This ratio was $13.8 \%$ in our study. Brain malformations are reported to be common in term born children, which was the case for our series. The results were both statistically and clinically significant. ${ }^{20-23}$

In a study by Truwit et $a .^{25}, 35 \%$ of CP patients were born preterm and according to MRI findings, perinatal brain damage was observed in only $24 \%$ of term CP patients, and $\mathrm{CP}$ was associated with prenatal causes, rather than a perinatal event. The percentage of children with $\mathrm{CP}$ born before the 37 th week was reported to be $25-40 \%$, while this rate was $29 \%$ in our series. Of CP cases in our study $66.4 \%$ were born at term and prenatal causes accounted for only $19.6 \%$ of the cases. In Nelson and Ellenberg's study, prenatal risk factors accounted for $70-80 \%$ of term infants that developed $\mathrm{CP}$, whereas in our study, the patients either had natal $(21.5 \%)$ or postnatal $(12.7 \%)$ or a combination of prenatal and postnatal causes $(37.5 \%)$, and the proportion of prenatal risk was low. This may be ascribed to low preterm birth rate $(29 \%) .^{24,25}$ This information can help us gain insights into the pathogenesis of $\mathrm{CP}$ and ultimately develop strategies for possible measures.

In conclusion the adequate number of cases examined using MRI in specified radiology units by two radiologists provided useful scientific data particularly on identifying the prognosis of cases, ensuring the management of clinical course, and determining test and treatment protocols.

\section{REFERENCES}

1. Reddihough DS, Collins KJ. The epidemiology and causes of cerebral palsy. Aust J Physiother 2003; 49: 7-12.

2. Ashwal S, Russman BS, Blasco PA, et al; Quality Standards Subcommittee of the American Academy of Neurology; Practice Committee of the Child Neurology Society. Practice Parameter: Diagnostic assessment of the child with cerebral palsy: Report of the Quality Standards Subcommittee of the American Academy of Neurology and the Practice Committee of the Child Neurology Society. Am Acad Neurol (AAN) 2004; 62: 851-863.

3. Rethlefsen SA, Ryan DD, Kay RM. Classification systems in cerebral palsy. Orthop Clin North Am 2010; 41: 457-467.

4. Paneth N. Establishing the diagnosis of cerebral palsy. Clin Obstet Gynecol 2008; 51: 742-748.

5. Jones MW, Morgan E, Shelton JE, Thorogood C. Cerebral palsy: introduction and diagnosis. J Pediatr Health Care 2007; 21: 146-152.

6. Johnston MV, Hoon AH Jr. Cerebral palsy. Neuromolecular Med 2006; 8: 435-450.

7. Shevell M, Miller SP, Scherer SW, Yager JY, Fehlings MG. The cerebral palsy demonstration project: A multidimensional research approach to cerebral palsy. Semin Pediatr Neurol 2011; 18: 31-39.

8. Johnston MV, Hagberg H. Sex and the pathogenesis of cerebral palsy. Dev Med Child Neurol 2007; 49: 74-78.

9. Legault G,Shevell MI, Dagenais L; Quebec Cerebral Palsy Registry Consortium. Predicting comorbidities with neuroimaging in children with cerebral palsy. Pediatr Neurol 2011; 45: 229-232.

10. Korzeniewski SJ, Birbeck G, Delano MC, Potchen MJ, Paneth N. A systematic review of neuroimaging for cerebral palsy. J Child Neurol 2008; 23: 216-227.

11. Krägeloch-Mann I. Understanding causation of cerebral palsy by using magnetic resonance imaging. Paediatr Child Health 2008; 18: 399-404.

12. Krägeloch-Mann I, Horber V. The role of magnetic resonance imaging in elucidating the pathogenesis of cerebral palsy: a systematic review. Dev Med Child Neurol 2007; 49: 144- 151.

13. Himmelmann K, Horber V, De La Cruz J, et al; SCPE Working Group. MRI classification system(MRICS) for children with cerebral palsy: development, reliability and recommendations. Dev Med Child Neurol 2017; 59: 57-64.

14. Tollånes MC, Wilcox AJ, Lie RT, Moster D. Familial risk of cerebral palsy: population based cohort study. Research. BMJ 2014; 349: 1-8. 
15. Moreno-De-Luca A, Ledbetter DH, Martin CL. Genomic insights into the etiology and classification of the cerebral palsies Lancet Neurol 2012; 11: 283 292.

16. Shorvon S. MRI of cortical dysgenesis. Epilepsia 1997; 38(Suppl 10): 13-18.

17. Zafeiriou DI, Kontopoulos EE, Tsikoulas I. Characterictics and prognosis of epilepsy in children with cerebral palsy. J Child Neurol 1999; 14: 289-294.

18. Sellier E, Uldall P, Calado E, et al. Epilepsy and cerebral palsy: Characterictics and trends in children born in 1976-1998. Eur J Paediatr Neurol 2012; 16: 48-55.

19. Krägeloch-Mann I, Cans C. Cerebral palsy update. Brain Dev 2009; 31: 537-544.

20. Accardo J, Kammann H, Hoon AH Jr. Neuroimaging in cerebral palsy. J Pediatr 2004; 145(Suppl 2): S19-S27.
21. Yin R, Reddihough DS, Ditchfield MR, Collins KJ Magnetic resonance imaging findings in cerebral palsy. J. Paediatr Child Health 2000; 36: 139-144.

22. Towsley K,Shevell MI, Dagenais L; REPACQ Consortium. Population-based study of neuroimaging findings in children with cerebral palsy. Eur J Paediatr Neurol 2011; 5: 29-35.

23. Bax M, Tydeman C, Flodmark O. Clinical and MRI correlates of cerebral palsy. The European Cerebral Palsy Study. JAMA 2006; 296: 1602-1608.

24. Robinson MN, Peake LJ, Ditchfield MR, Reid SM, Lanigan A, Reddihough DS. Magnetic resonance imaging findings in a population-based cohort of children with cerebral palsy. Dev Med Child Neurol 2008; 51: 39-45.

25. Truwit CL, Barkovich AJ, Koch TK, Ferriero DM. Cerebral palsy: MR findings in 40 patients. AJNR Am J Neuroradiol 1992; 13: 67-78. 